\title{
SPRINGBACK COMPENSATION IN SHEET METAL FORMING USING A SUCCESSIVE RESPONSE SURFACE METHOD
}

\author{
Nielen Stander, Mike Burger, Xinhai Zhu and Bradley Maker \\ Livermore Software Technology Corporation, 7374 Las Positas Road, Livermore, CA \\ Email: nielen@1stc.com,mike@1stc.com, zhu@1stc.com, maker@1stc.com
}

\begin{abstract}
The purpose of this study is to demonstrate a methodology for springback compensation in sheet metal stamping operations. An optimization method based on the $D$-optimality criterion for experimental design and successive linear response surface approximations is employed to minimize the difference between the simulation results and the intended design. A subdomain reduction scheme is applied to determine the subdomain size for each iteration. The procedure, which has been implemented in the program LS-OPT, is interfaced with the simulation package LS-DYNA, and the parametric preprocessor TrueGrid. These tools are used to input original tool geometry, material, and process parameters, identify design variables, perform springback simulations and output optimized tool geometry. The standardized NUMISHEET'96 S-Rail is used as a benchmark example in this study. A converged optimized design is obtained in four or five iterations. It is found that springback trends are consistent with changes in the die shape, thereby suggesting other effective strategies for springback compensation.
\end{abstract}

\section{Introduction}

Springback is an elastic deformation which occurs at the end of a sheet metal stamping process, as the stamped part is removed from the stamping tools. This phenomenon has the effect of changing the finished shape of the part so that it no longer matches the forming tools. If this shape deviation is large, it can cause difficulty during a subsequent assembly process, or render the assembled part unusable. Accordingly, it is important to produce parts of which the finished shapes closely match the designed surface. Usually

Copyright (C) 2002 by Livermore Software Technology Corporation. Published by the American Institute of Aeronautics and Astronautics, Inc., with permission. corrections to compensate for springback are made by modifying the shape of the stamping tools.

Before the computer simulation became acceptable, trial-and-error methods were used for springback compensation in industry. This approach requires many years of die-shop experience and is also very time consuming - to make a modified die set could take months. In addition, several trial-and-error corrections are frequently required before adequately compensated parts are obtained. Accordingly, the trial-and-error process is very expensive, often requiring on the order of a million dollars to make a die that produces "good" parts. When new materials are used or when a new design is adopted, previous experience cannot be applied directly. The difficulties associated with the trial-and-error method can result in uncertainty in predicting costs and lead-times.

Computer simulation has gained popularity in the stamping industry due to its speed and low cost, and it has been proven to be effective in prediction of formability and springback behavior ${ }^{2}$. However, no effective direct method is available to modify the die based on the predicted springback. Rather, an inverse analysis requiring an iterative scheme seems to be a requirement. Despite this drawback, approximate methods exist which can, in some cases, improve the tool design.

The spring-forward method is based on numerical simulation by Finite Element Analysis (FEA). The method begins by performing a stamping simulation, from which information for the deformed part is obtained while it is still positioned in the closed dies. This information includes the geometry, and material stress and strain data. The method then assumes that subsequent springback deformation will be driven by material stress, and that if the stress distribution through the material thickness is reversed, the resulting springback deformation will also be in the reversed 
direction, as compared to the actual part. Based on this logic, the geometry, which is obtained by springback analysis with reversed stress, can be used to predict modifications to the dies. This method is very simple to apply, and it is the most popular numerical method. However, the method suffers from two major shortcomings that prohibit use in many practical applications. These are under-cut (interference between the parts during the stamping process) and accuracy.

The purpose of this study is therefore to investigate an effective iterative algorithm for springback compensation. An optimization method is applied to solve the inverse problem. Several component tools are required. TrueGrid ${ }^{9}$ is used as a pre-processor to parametrically define geometry of the rigid tools, LSDYNA $^{4}$ software is used as a Finite Element based solver and LS-OPT ${ }^{6}$ is used to guide the solution to an optimum. The NUMISHEET'96 S-RAIL ${ }^{1}$ example is used as a benchmark.

\section{Approach}

The die compensation analysis presented here uses an optimization method based on an iterative response surface scheme. Many stamping/springback simulations are performed during the iterative process. Using the parametric preprocessor TrueGrid, the user can introduce a set of design variables. These may include geometric variables, such as critical locations where tool elevation should be modified, important tool radii, and starting blank geometry. Process variables may also be specified, such as drawbead restraining forces and binder and pad loads.

Using this design variable information, LS-OPT automatically creates a set of stamping/springback models (according to an experimental design method), and submit several simultaneous simulation jobs. These jobs are distributed through a computer network since they execute independently and will therefore exhibit near perfect parallel efficiency.

After collecting and processing results from the first set of simulations, LS-OPT predicts optimized values for each design variable, using a response surface based on the result set for each response. Using these optimized design variables, the next set of simulations (experimental design) is automatically created and submitted. This iterative process continues until each variable has been determined within a specified tolerance, or until a limiting number of iterations have been completed.

\section{Methodology}

Consider the general nonlinear optimization problem:

Minimize

$$
f(\boldsymbol{x}), \boldsymbol{x} \in R^{n}
$$

subject to the inequality constraints

$$
L_{j} \leq g_{j}(\boldsymbol{x}) \leq U_{j} ; j=1,2, \ldots, m
$$

and simple bounds on the design variables

$$
x_{i l} \leq x_{i} \leq x_{i u} ; i=1, \ldots, n
$$

where $L_{j}$ and $U_{j}$ refer to the upper and lower bounds on each of the inequality constraints, and $x_{i l}$ and $x_{i u}$ the lower and upper bounds on each of the design variables, $n$ is the number of design variables, and $m$ the number of inequality constraints. Note that equality constraints can be written as two inequality constraints in the form of Equation 2 with $L_{j}$ equal to $U_{j}$.

References 5, 7 and 8 can be consulted for a detail description of the Successive Response Surface Method (SRSM). The method, as implemented in LS-OPT $^{5}$ contains a number of features that makes it robust and suitable for the solution of practical problems:

- The $D$-optimal experimental design is used to best utilize the number of available runs. Oversampling of $50 \%^{5}$ is used to maximize the predictive capability of the response surfaces.

- Linear approximations are constructed using linear regression on all the points of the current iteration. Unit weighting is used for the regression.

- An adaptive domain reduction $\operatorname{method}^{8}$ is applied as described below.

- An auxiliary problem that minimizes the maximum constraint violation ${ }^{6}$ is solved to enforce feasible designs. This formulation is effective in minimizing maximum discrepancies such as those resulting from springback.

The SRSM method uses a region of interest, a subspace of the design space, to determine an approximate optimum. A range is chosen for each variable to determine its initial size. A new region of interest centers on each successive optimum. Progress is made by moving the center of the region of interest as well as reducing its size. Figure 1 shows the possible adaptation of the subregion. 
The starting point $\boldsymbol{x}^{(0)}$ will form the center point of the first region of interest. The lower and upper bounds $\left(x_{i}^{r L, 0}, x_{i}^{r R, 0}\right)$ of the initial subregion are calculated using the specified initial range value $r_{i}^{(0)}$ so that

$$
\begin{aligned}
& x_{i}^{r L, 0}=x_{i}^{(0)}-0.5 r_{i}^{(0)} \\
& x_{i}^{r U, 0}=x_{i}^{(0)}+0.5 r_{i}^{(0)} i=1, \ldots . n
\end{aligned}
$$

where $n$ is the number of design variables. The modification of the ranges on the variables for the next iteration depends on the oscillatory nature of the solution and the accuracy of the current optimum.

A contraction parameter $\gamma$ is firstly determined based on whether the current and previous designs $\boldsymbol{x}^{(k)}$ and $\boldsymbol{x}^{(k-1)}$ are on the opposite or the same side of the region of interest. Thus an oscillation indicator $c$ may be determined in iteration $k$ as

$$
c_{i}^{(k)}=d_{i}^{(k)} d_{i}^{(k-1)}
$$

where

$$
\begin{aligned}
& d_{i}^{(k)}=2 \Delta x_{i}^{(k)} / r_{i}^{(k)} ; \\
& \Delta x_{i}^{(k)}=x_{i}^{(k)}-x_{i}^{(k-1)} ; \\
& d_{i}^{(k)} \in[-1 ; 1]
\end{aligned}
$$

The oscillation indicator (purposely omitting indices $i$ and $k$ ) is normalized as $\hat{c}$ where

$$
\hat{c}=\sqrt{|c|} \operatorname{sign}(c) .
$$

The contraction parameter $\gamma$ is then calculated as

$$
\gamma=\frac{\gamma_{\mathrm{pan}}(1+\hat{c})+\gamma_{\mathrm{osc}}(1-\hat{c})}{2} .
$$

The parameter $\gamma_{\text {osc }}$ is typically $0.5-0.7$ representing shrinkage to dampen oscillation, whereas $\gamma_{\text {pan }}$ represents the pure panning case and therefore unity is typically chosen.

The accuracy is estimated using the proximity of the predicted optimum of the current iteration to the starting (previous) design. The smaller the distance between the starting and optimum designs, the more rapidly the region of interest will diminish in size. If the solution is on the bound of the region of interest, the optimal point is estimated to be beyond the region.
Therefore a new subregion, which is centered on the current point, does not change its size. This is called panning (Figure 1(a)). If the optimum point coincides with the previous one, the subregion is stationary, but reduces its size (zooming) (Figure 1(b)). Both panning and zooming may occur if there is partial movement (Figure 1(c)). The range $r_{i}^{(k+1)}$ for the new subregion in the $(k+1)$-th iteration is then determined by:

$$
\begin{aligned}
& r_{i}^{(k+1)}=\lambda_{i} r_{i}^{(k)} ; \\
& i=1, \ldots, n ; k=0, \ldots, n i t e r
\end{aligned}
$$

where $\lambda_{i}$ represents the contraction rate for each design variable. To determine $\lambda_{i}, d_{i}^{(k)}$ is incorporated by scaling according to a zoom parameter $\eta$, typically 0.5 , that represents pure zooming and the contraction parameter $\gamma$ to yield the contraction rate

$$
\lambda_{i}=\eta+d_{i}^{(k)} \mid(\gamma-\eta)
$$

for each variable independently (see Figure 2).

\section{Example: Numisheet 96 S-Rail}

The tools and sheet-metal blank of the Numisheet 96 springback benchmark ${ }^{1}$ problem are shown in Figure 3. The punch is controlled at a constant $1 \mathrm{~m} / \mathrm{s}$ while the binder is driven by a piece-wise linear force curve as shown in Fig. 4.

Formulation of the optimization problem

The objective of the design procedure is to maximize the flatness of the flange pair as if the work-piece were to be welded to a flat surface. To achieve this, a flat surface is fitted through 24 selected flange points, using a linear regression analysis. These points are selected at the flange inner and outer positions as shown in Figure 5. The offset of a point can be computed as $e_{i}=z_{i}$ $Z_{i}$ where $z_{i}$ is the vertical coordinate of the point $i$ and $Z_{i}$ is the vertical position of the point projected on the plane. Using the selected points, two possible main approaches are available to formulate the design problem.

1. RMS: Compute a root mean square (RMS) residual of the perpendicular offset of each point on the work-piece after springback, $\sqrt{\sum_{i=1}^{24} e_{i}^{2} / 24}$, and use it as the objective for minimization. 
2. Maximum: Constrain the offset of each point: $-E \leq e_{i} \leq E, \quad i=1,2, \ldots 24$ and minimize the auxiliary variable $E$, keeping $E>0$. The effect of this formulation is to minimize the greatest offset after springback.

\section{Design variables}

Nine design variables were chosen, namely the radius $r$ which applies to all four corners of the cross-section as well as the positions of 8 control points on the outer perimeter of the die and binders. The model was parameterized with TrueGrid. The control points are connected by straight lines to hinge points at the tangent line to the radius. The control points define the tool surfaces by controlling the $z$-coordinates of the selected points as shown in Figure 6. For the baseline design all the $x$ values are zero.

Note that the binder has been parameterized to assume the same shape as the die for a snug fit of the tools. At $t=0$ the binders and die have to be sufficiently spaced to prevent interference with the sheet metal blank (see Figs. 6 and 8). This condition is formulated in the TrueGrid input file.

Simple bounds have been chosen for the variables so that the optimization problem formulation becomes:

\section{$\operatorname{Min} E$}

subject to

$$
\begin{gathered}
-E \leq e_{i} \leq E ; i=1,2, \ldots 24 \\
-25 \mathrm{~mm}<x_{j}<25 \mathrm{~mm} ; \quad j=1,2, \ldots 8 \\
3 \mathrm{~mm}<R<7 \mathrm{~mm}
\end{gathered}
$$

where $R$ is the corner radius.

\section{$\underline{\text { Results }}$}

LS-OPT, employing Formulation 2 (maximum offset) was used to optimize the tool design. The problem was run on an HP V-class 16 processor server. 8 processors were utilized. 16 simulations were conducted per iteration. The time required for a full iteration is 3.5 hours. About 5 to 6 iterations were required for convergence. Further iterations were run to attempt finer convergence, but the maximum offset remained at $\sim 0.8 \mathrm{~mm}$ compared to the baseline $3.2 \mathrm{~mm}$ (Figure $7 \mathrm{a}$ ).

The dots represent the simulated results using LSDYNA, whereas the line represents the response surface prediction. The history of variable $x_{8}$ (lower and upper bounded line in Figure 7b) suggests convergence by iteration 5 . Note that the optimal flange shape is very close to a flat surface (Figs. 8a, b and c). Figure 9 shows the optimal tool shape that will minimize the surface warp.

\section{Conclusions}

From the above benchmark, we can draw the following conclusions:

- As with all optimization procedures, the effectiveness of the method for springback compensation relies on the choice of a sufficient number of suitable design variables. Even with the choice of nine variables, the springback could only be reduced by about $70 \%$.

- Using TrueGrid, the surfaces are mathematically defined (not simply a mesh perturbation), and therefore the parametrization can be designed in accordance with manufacturing requirements.

- Judging by the optimum die geometry, the springback behavior is consistent with small to moderate perturbations in tool geometry. I.e. even with the change of the tool geometry, the springback still occurs in the same direction as before.

Drawbacks of the optimization method are as follows:

- The choice of design variables depends heavily on the user's experience, which makes it difficult for complex part design. With more powerful hardware and software it is conceivable that, initially, a large number of variables can be chosen from which a suitable subset can be selected by means of variable screening (for instance using an analysis of variance (ANOVA) $\left.\operatorname{method}^{3}\right)$.

- Optimization is expensive in terms of simulation time. Large, realistic sheet metal forming models typically have run times of days rather than hours.

In spite of the drawbacks, the results are encouraging in terms of the accuracy of the results obtained and the robustness of the method. In the mean time other, mesh-based methods are being investigated as a means to accelerate the optimization phase of the procedure by reducing the number of variables. 


\section{$\underline{\text { References }}$}

1. Anon. S-Rail Benchmark Problem (1996). Proceedings of the $3^{\text {rd }}$ International Conference: Numerical Simulation of 3-D Sheet-Metal Forming Processes - Verification of Simulations with Experiments (NUMISHEET '96). Eds. Lee, Kinzel and Wagoner, September, 1996, pp. 612-799.

2. He, N. and Wagoner, R.H (1996). Springback Simulation in Sheet Metal Forming. Proceedings of the $3^{\text {rd }}$ International Conference: Numerical Simulation of 3-D Sheet-Metal Forming Processes - Verification of Simulations with Experiments (NUMISHEET '96). Eds. Lee, Kinzel and Wagoner, September, 1996, pp. 308-315.

3. Khuri, A.I. and Cornell, J.A. (1996) Response Surfaces, Designs and Analyses. Marcel Dekker, Inc., New York. p.43.
4. Livermore Software Technology Corporation (2001), LS-DYNA User's Manual, Version 960.

5. Roux, W.J., Stander, N., and Haftka, R.T. (1998), "Response Surface Approximations for Structural Optimization", International Journal for Numerical Methods in Engineering, 42, 517-534.

6. Stander, N. (1999), LS-OPT User's Manual Version 1, Livermore Software Technology Corporation, Livermore, CA.

7. Stander, N. (2001) "The Successive Response Surface Method Applied to Sheet-Metal Forming", Proceedings of the First MIT Conference on Computational Fluid and Solid Mechanics, Boston, June 12-14, 2001. Elsevier Science Ltd., Oxford.

8. Stander N, Craig KJ (2002). On the robustness of a domain reduction scheme for simulation-based optimization. To appear. Engineering Computations. 2002. 19(4).

9. TrueGrid manual (2000), XYZ Scientific Applications, Inc. Livermore, CA.

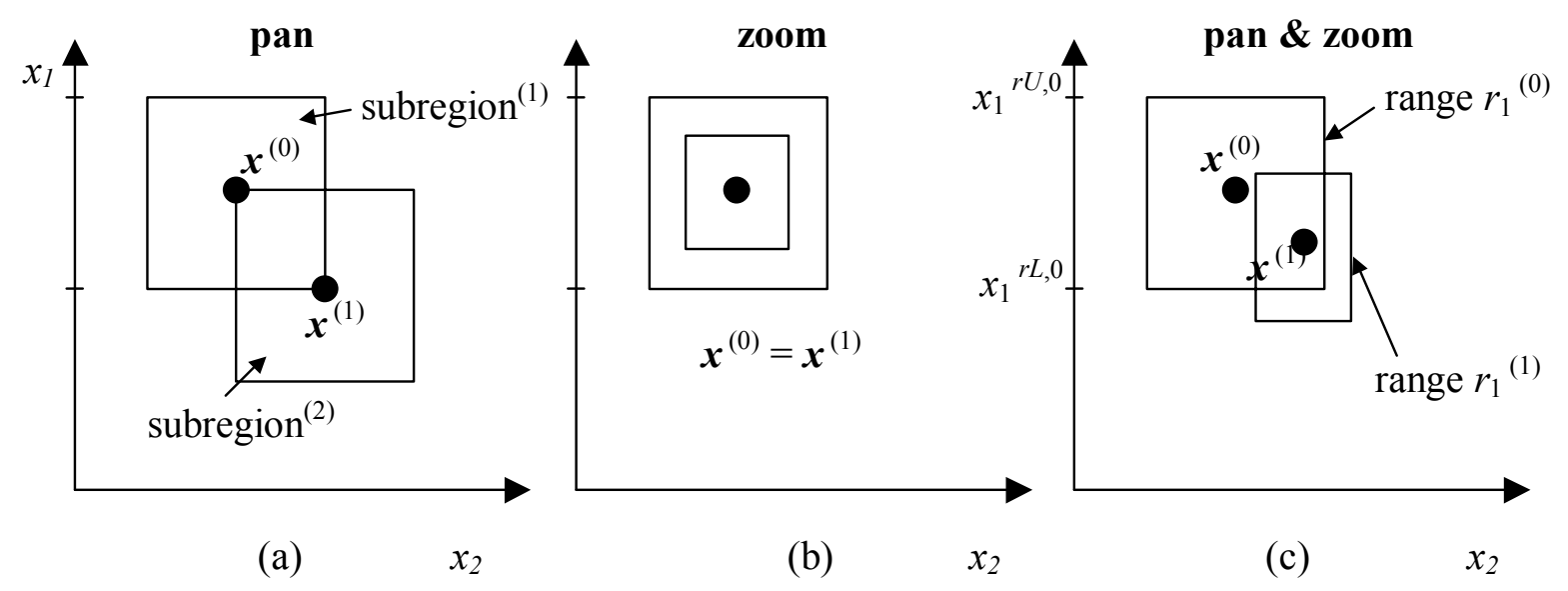

Figure 1: Adaptation of subregion in SRSM: (a) pure panning, (b) pure zooming and (c) a combination of panning and zooming 


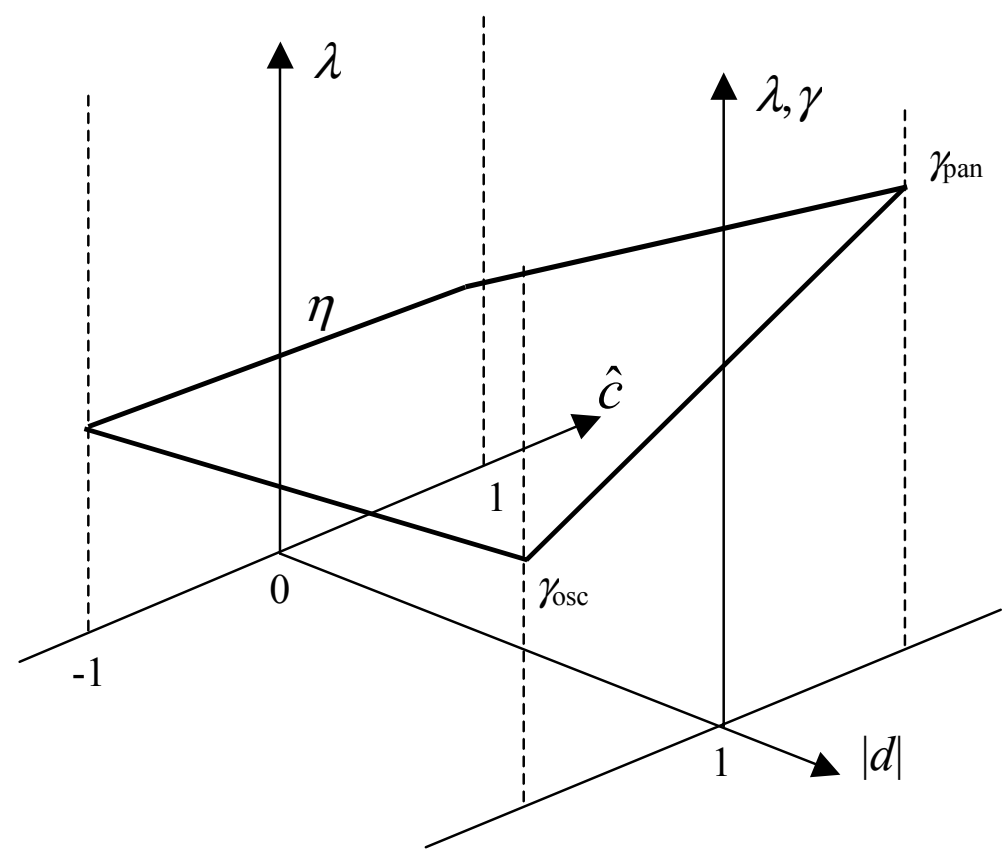

Figure 2: Domain reduction scheme: the sub-region contraction rate $\lambda$ as a function of the oscillation indicator $\hat{c}$ and the absolute move distance $|d|$

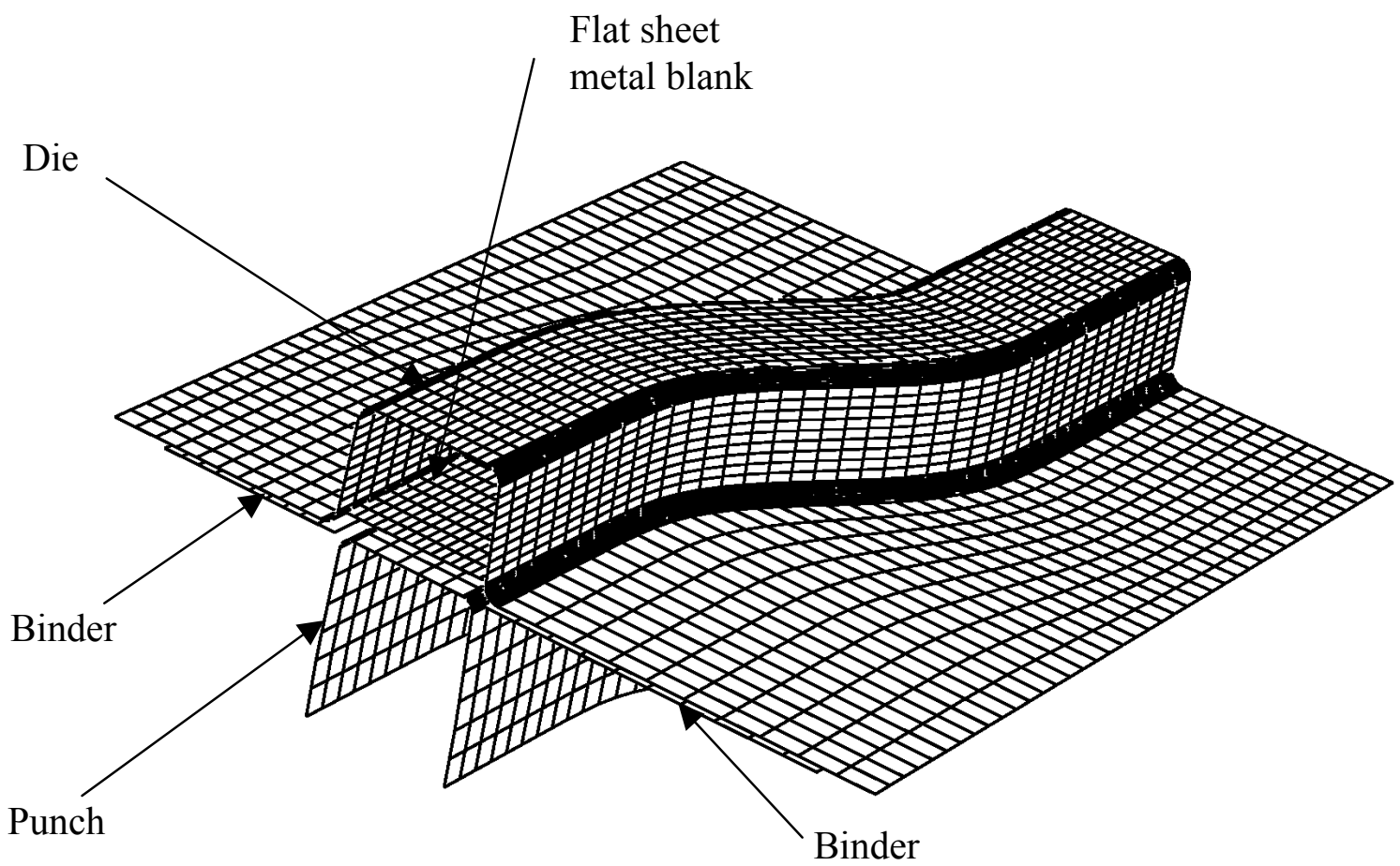

Figure 3: Numisheet 96 benchmark: Punch, die, binders and blank (baseline design) 


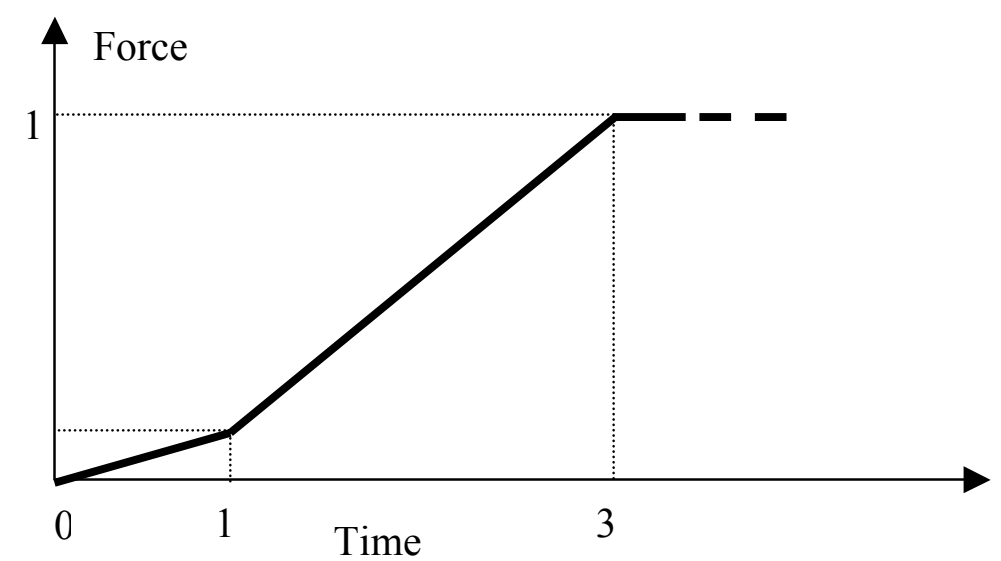

Figure 4: Binder Force as a function of time

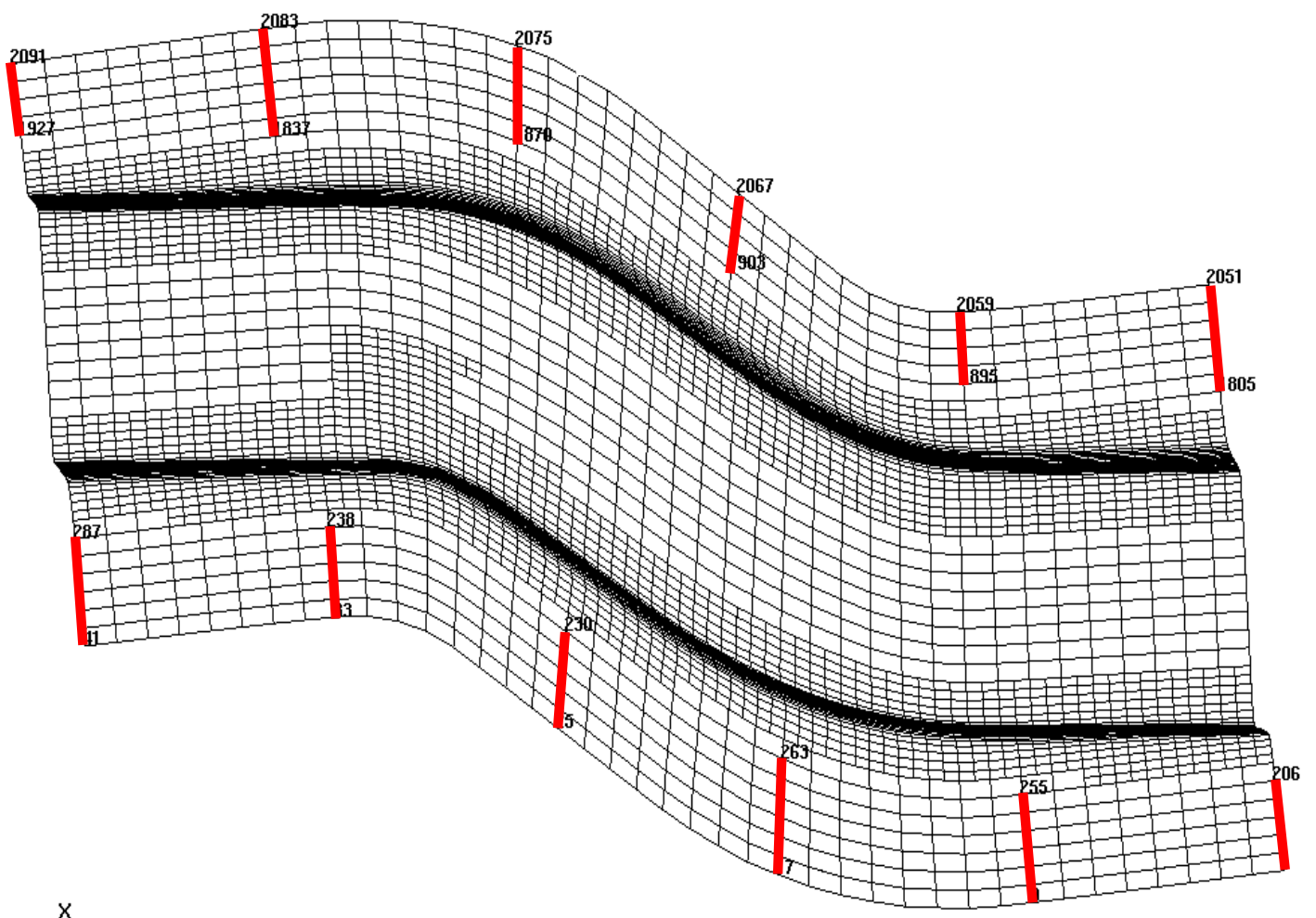

Figure 5: Monitoring points on flanges (top view) 


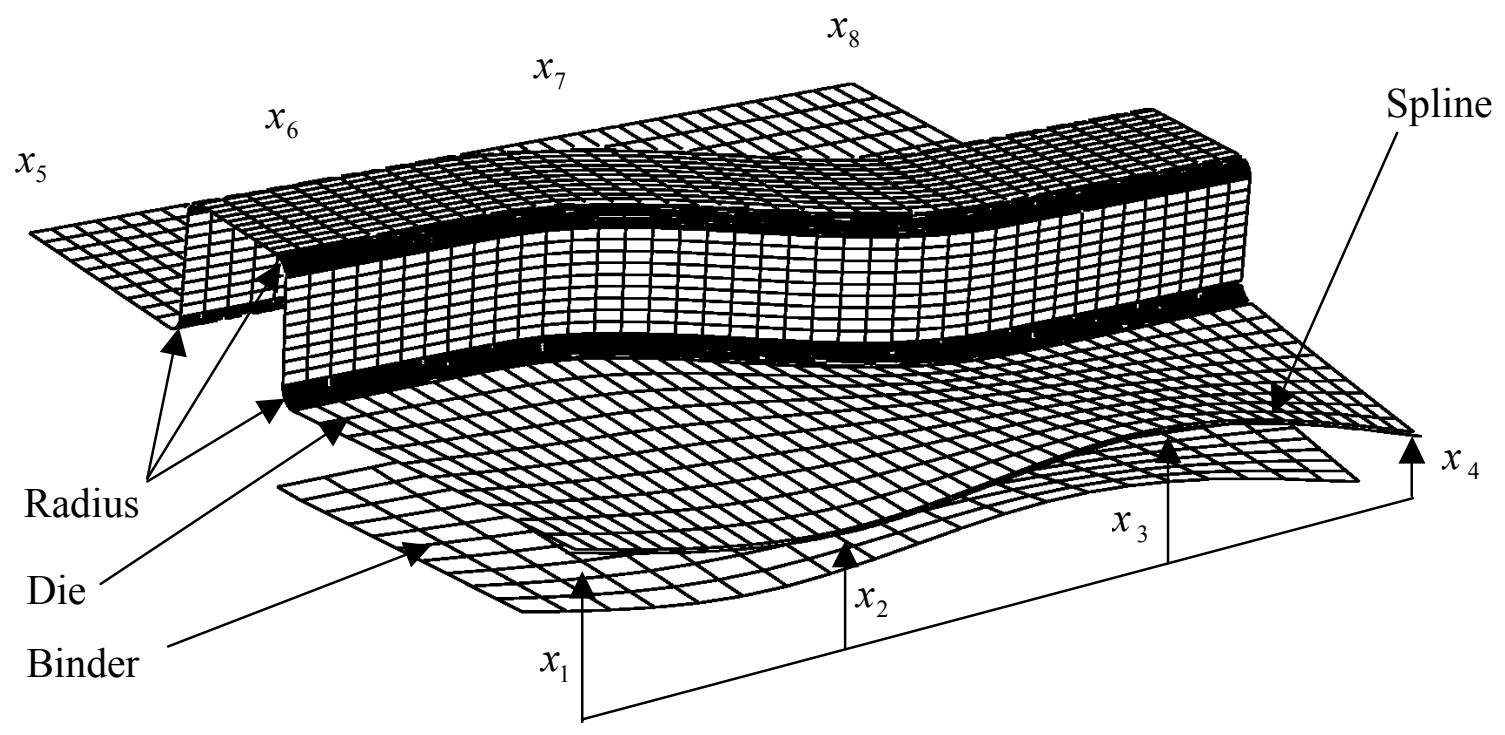

Figure 6: Design variables ( $x_{1}$ to $x_{8}$ and Radius)
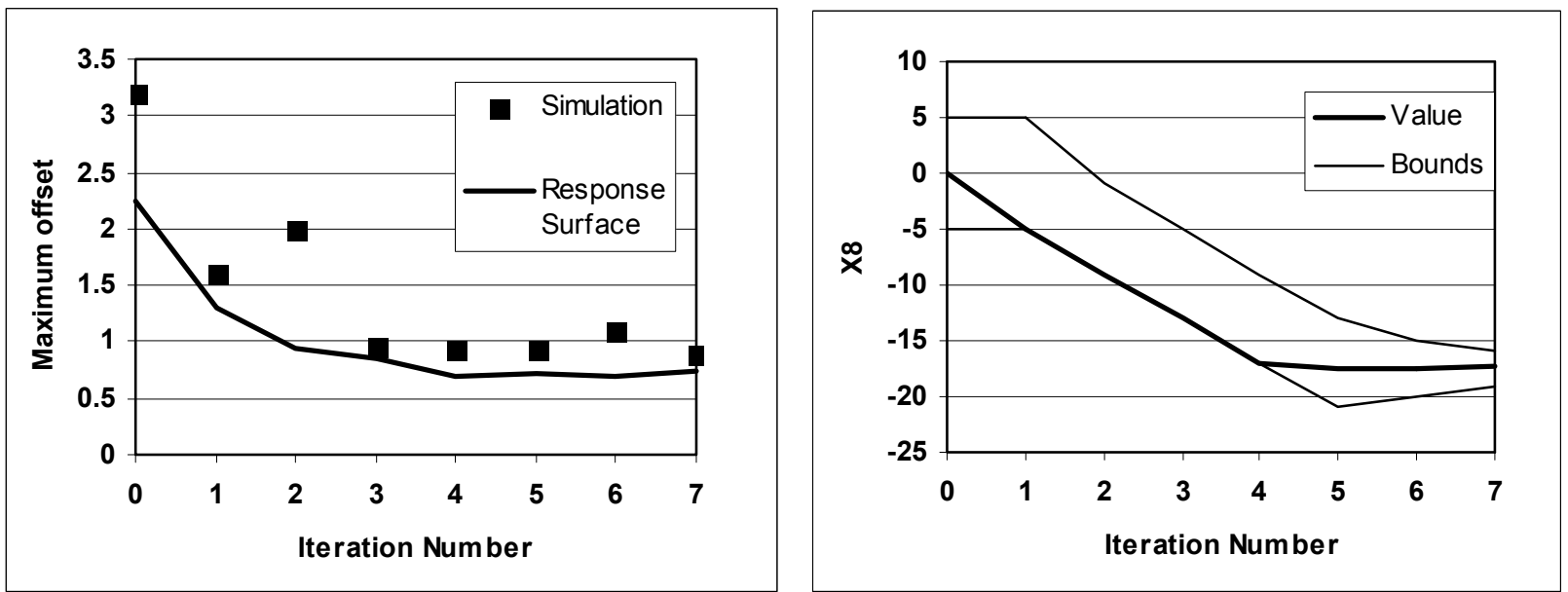

Figure 7: Optimization history of (a) maximum offset and (b) variable $x_{8}$ 


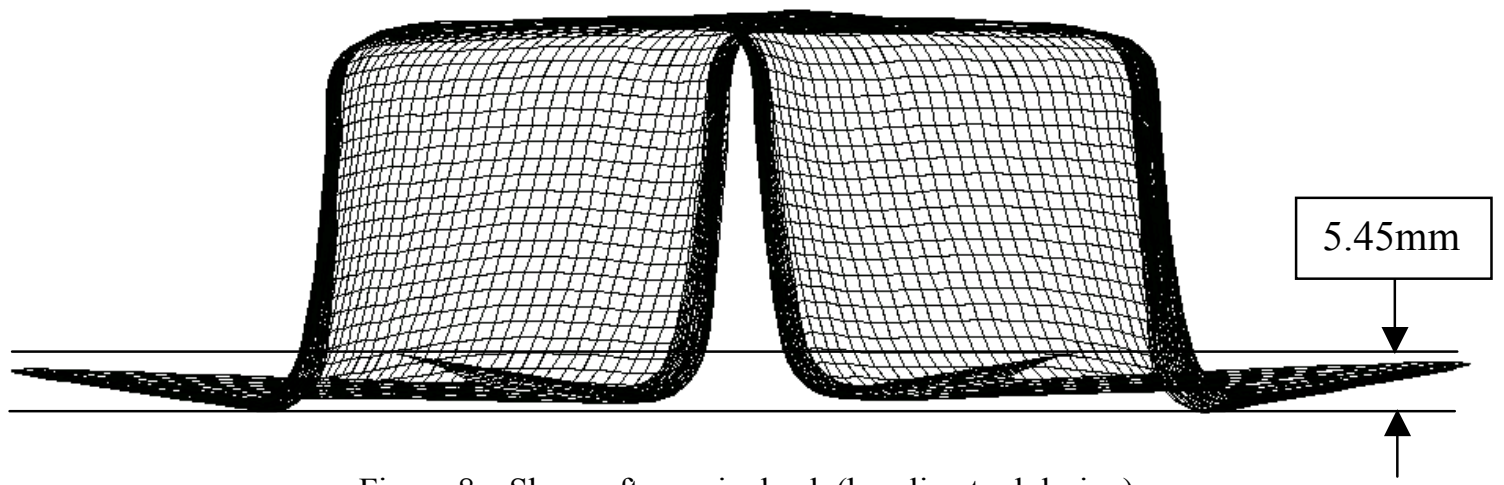

Figure 8a: Shape after springback (baseline tool design)

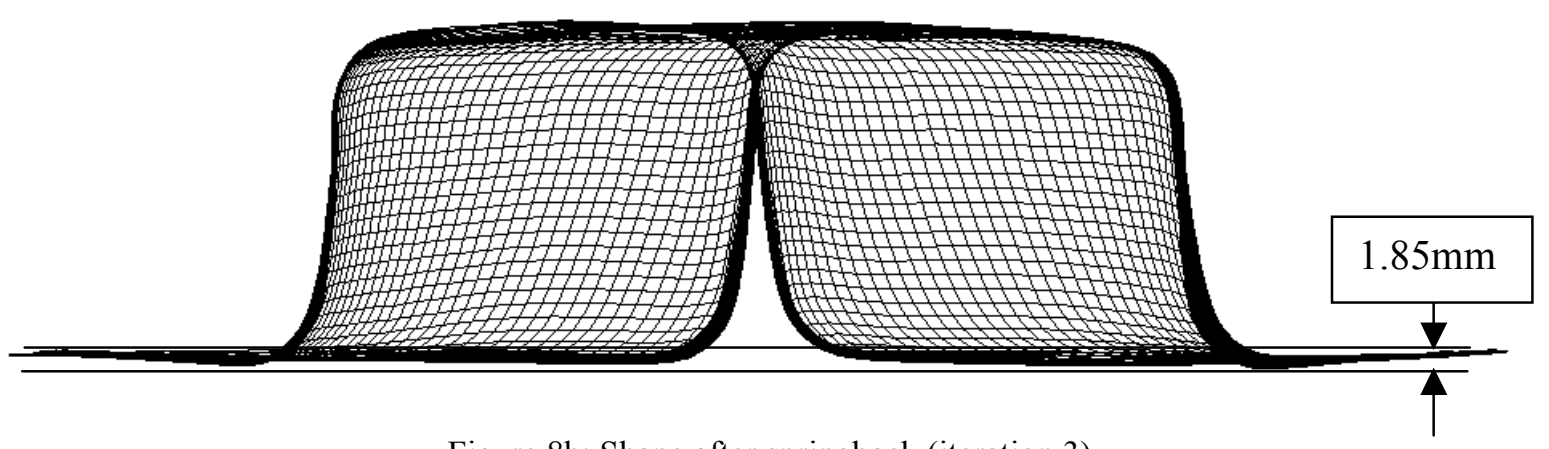

Figure 8b: Shape after springback (iteration 3)

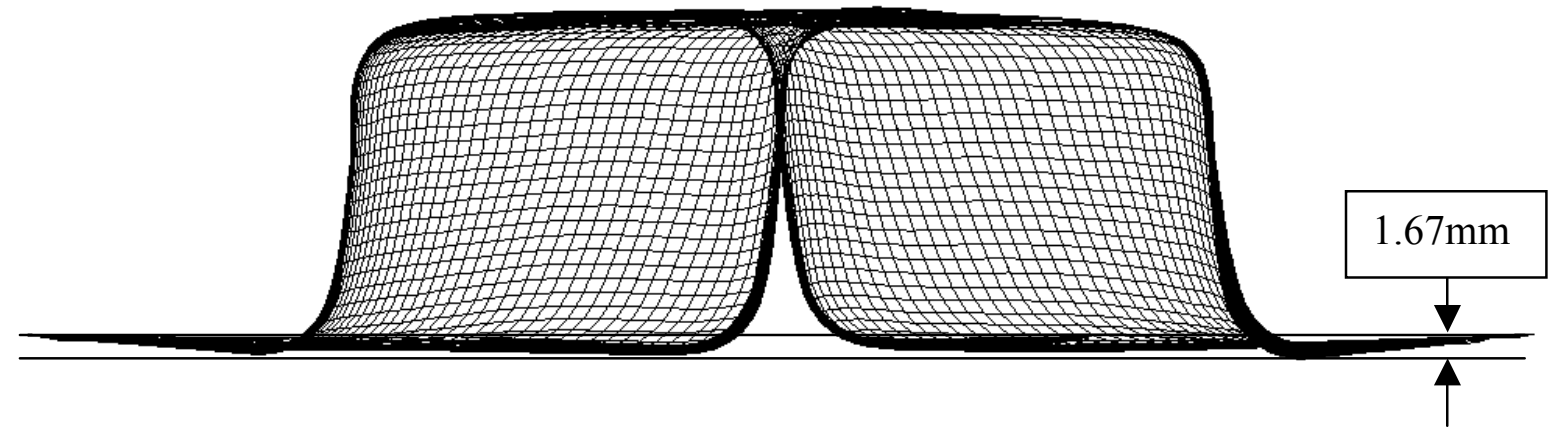

Figure 8c: Shape after springback (iteration 10) 


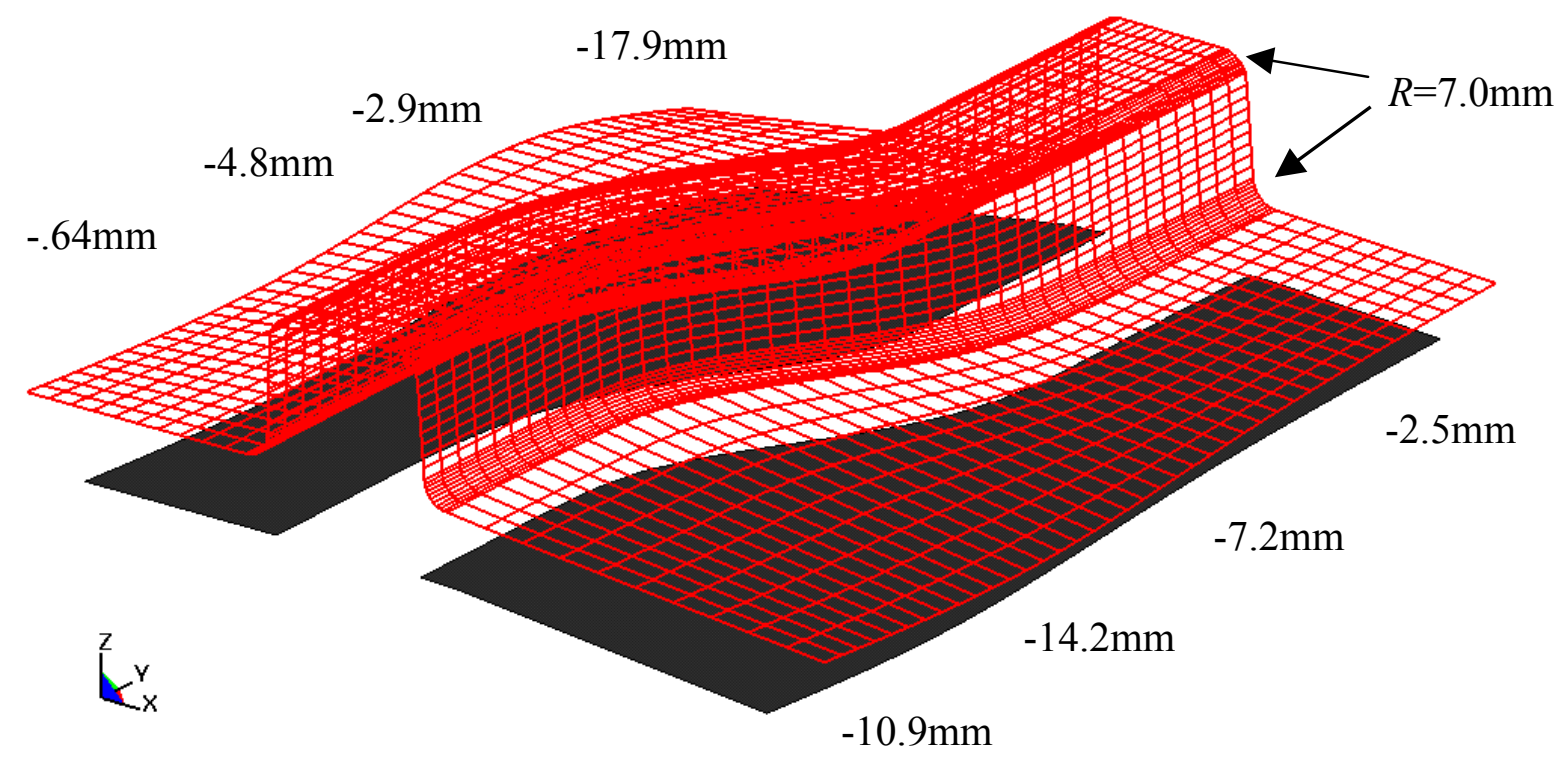

Fig. 9: Optimum die geometry (iteration 10) 